\title{
Editor's Message to Special Issue of Usable Security
}

\author{
KATSUMI TAKAHASHI $^{1, \text { a }}$
}

We have finished editing the special issue of "Usable Security" and I would like to give a background and an acknowledgement on behalf of the editorial committee of this issue.

We planned this special issue in early 2018 and formed 25 members of editorial committee. We announced the call for paper in October 2018. After five months of submission period, we have received 29 submissions and we spent six months for review process. Finally, 20 papers are accepted. Acceptance rate is $69 \%$ and four English papers are included.

The simple reason why we focused on Usable Security is the importance of the security. Security is necessary for from our daily life like smart phones or Web to the social infrastructure. The key for the achievement of security is the existence of human. It is obvious that usability helps the security and the vulnerability could be brought by the human, but highly advanced systems become, more important these characteristics are.

In this issue, we describe Usable Security as security and privacy studies considering usage of human. Security is for information security and the privacy enhancing technologies are included.

Usable Security has been studies in SIG SPT -Security Psychology and Trust- of IPSJ and SPT has started a workshop named Usable Security Workshop since 2017. We set the scope wide in this issue, because we do not regard Usable Security established but regard promising for contributors of this area. Scope included network security, computer security, data privacy, studies for usability and efficiency, studies for operations, studies against cyber-attacks which abuse the legitimate use, studies of human related errors, and consent and ethics.

We have discussed our reviewing policy, reminded the IPSJ's one "Even if you pick up pebbles, do not throw gemstones away" and asked reviewers to evaluate the contribution of the article even if it is small. We also asked them to confirm authors how "usable" their proposal is for security. As we requested reviewers to many efforts, it brought the $69 \%$ of acceptance.

Major category of accepted papers is the authentication (including biometrics) and six papers can be found. Papers for usability and privacy also characterize this issue. Papers for risk management or evaluations are filed under information security management. Papers for network security and operation system security are also included. You can find usable techniques and practices.

We believe that Usable Security will be studied more and helps real world's security and privacy continuously. We hope IPSJ keeps encouraging this area and another special issue of Usable Security will be published in the next year.

We would like to thank IPSJ editorial committee and SPT members for giving us to edit this special issue. We thank to editorial committee for this special issue for discussion and high quality review. Also, we thank to reviewers for supporting the authors. We believe this issue helps encouraging the security of computer systems around us.

\section{The Editorial Committee}

- Editor in-Chief:

Katsumi Takahashi (NTT Secure Platform Laboratories).

- Editorial Board Members:

Masue Shiba (Toshiba Solutions)

Masaki Shimaoka (SECOM)

\section{- Editorial Committee Members:}

Masatsugu Ichino (University of Electro-Communications)

Atsuo Inomata (Osaka University)

Midori Inaba (Institute of Information Security)

Tetsutaro Uehara (Ritsumeikan University)

Satoshi Ozahata (University of Electro-Communications)

Akira Kanaoka (Toho University)

Ayako Komatsu (University of Nagasaki)

Hidehito Gomi (Yahoo Japan)

Takahito Sakamoto (SECOM)

Yoshiaki Shiraishi (Kobe University)

Tetsuji Takada (University of Electro-Communications)

Kenji Tanaka (University of Electro-Communications)

Toshiaki Tanaka (KDDI R\&D Labs)

Yukiyasu Tsunoo (Tokyo Online University)

Masato Terada (Hitachi)

Masakatsu Nishigaki (Shizuoka University)

Keiichi Hirota (NTT)

Kanta Matsuura (University of Tokyo)

Yuko Murayama (Tsuda College)

Koichi Mouri (Ritsumeikan University)

Takayasu Yamaguchi (NTT DOCOMO)

Hiroshi Yoshiura (University of Electro-Communications)

\footnotetext{
NTT Secure Platform Laboratories
}

a) katsumi.takahashi.aw@ @co.ntt.co.jp 